\title{
DHT domain digital watermarking with low loss in image informations
}

\author{
Santi P. Maity ${ }^{\mathrm{a}, *}$, Malay K. Kundu ${ }^{\mathrm{b}}$ \\ a Department of Information Technology, Bengal Engineering and Science University, Shibpur, P.O. Botanic Garden, 711 103, India

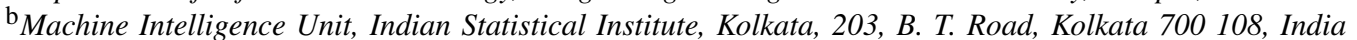

Received 25 May 2008; accepted 8 October 2008

\begin{abstract}
The paper proposes a digital image watermarking scheme that selects regions for data embedding based on information measure. Two valued kernels of Hadamard transformation cause smaller image information change during embedding compared to other transform domains such as DCT (discrete cosine transform), DFT (discrete Fourier transform), Fourier-Mellin and wavelet-based embedding. Moreover, the usage of Hadamard transform as signal decomposition tool offers advantages in terms of simpler implementation, low computation cost and high resiliency at low quality compression considering both JPEG and JPEG 2000 framework. Compression resiliency is further improved using adaptive negative modulation. These facts are validated comparing the performance with some other existing watermarking schemes as well as DCT domain implementation of the proposed scheme.
\end{abstract}

(C) 2009 Elsevier GmbH. All rights reserved.

Keywords: Digital image watermarking; Entropy; Hadamard transform; Negative modulation; Lossy compression

\section{Introduction}

Rapid growth of World Wide Web (WWW) has made possible for the owners of the digital medias to distribute their works on their web pages or in other public places. Anyone having access to those forums can copy those information as perfect duplicate of the originals due to the very nature of the digital media [1]. The question is, how does someone can claim the ownership of his/her creation in digital media when multiple persons can have the exact copies? One way to protect this ownership right is to embed additional information invisibly before the digital media is made available to the public. This embedded information is popularly known as digital watermark and it can provide, for example, information about the media, the author, copyright or licence, etc. [2,3].

\footnotetext{
* Corresponding author. Tel.: +913326684561; fax: +913326682916.

E-mail addresses: santipmaity@it.becs.ac.in (S.P. Maity), malay@isical.ac.in (M.K. Kundu).
}

Watermarking in digital images should possess data imperceptibility and robustness to several unintentional as well as deliberate attacks. To achieve robustness, data should be embedded in the regions that contain some important characteristics information of the cover image [4]. This is based on the basic assumption that so long as the important characteristics of the different regions in the cover image are not drastically changed, hidden data can be extracted faithfully. Some of the major characteristics of a gray level image are edge, texture and high gray level curvature points, etc. [5]. Robustness is further improved if watermark information is embedded in the suitable transform coefficients of the image characteristics.

Several digital image watermarking schemes using discrete Fourier transform (DFT) [6], Fourier-Mellin [7], discrete cosine transform (DCT) [8,9], and wavelet transformations $[9,10]$ have already been reported in the literature. Among them, DCT and wavelet transforms become popular as the most common image compression techniques e.g. JPEG and JPEG 2000 are either based on DCT or wavelet. 
However, it is found in the digital watermarking literature that most of the DCT domain embedding methods are very much robust to JPEG but are not similarly robust against JPEG 2000. The reverse is true for wavelet-based watermarking methods which are very much robust to JPEG 2000 compression operation but are not similarly robust against JPEG. So the choice of the proper decomposition tool is an important aspect to design a robust data hiding scheme when no knowledge about the compression scheme is available in advance. Ramkumar et al. [11] reported that Hadamard and Hartley transforms, due to the small values of the standard deviation for the processing noise, provide higher resiliency at low quality compression compared to DCT and wavelet transforms.

Discrete Hadamard transform (DHT) and its variants are used extensively to develop watermarking algorithms for multimedia signals. Li et al. [12] propose block-based DHT method where watermark information is inserted into Hadamard coefficients using quantization. The subblocks for watermark embedding are selected pseudo-randomly to increase security. Ho et al. [13] propose image-in-image watermarking method where DHT coefficients of gray scale watermark image modulate the DHT coefficients of the cover image. Image characteristics such as edges and textures are used to determine the watermark strength factor. Gilani et al. [14] propose multiresolution watermarking where image is first decomposed by means of single level Haar transform and Hadamard transform is then applied to the lowest frequency (LL) band only. Appropriate middle frequency coefficients are then selected through zigzag scanning and are used to embed watermark.

Multiresolution Hadamard transform is also used by Falkowski and Lim [15] where image is decomposed into a pyramid structure with various bands such as the low-low, low-high, high-high frequency band, etc. The lowest frequency band $(\mathrm{LFB})$ is then segmented into $(8 \times 8)$ blocks and the two-dimensional CHT (complex Hadamard transform) is applied next. The phase components of the selected coefficients are altered to convey the watermark information. Watermark recovery process requires both the original image and the watermark. Almost similar type of phase watermarking for the multimedia signals is proposed in [16] with two-level inverse difference pyramid decomposition where the coefficients are obtained with CHT. The algorithm claims that the two-level pyramid decomposition permits the insertion of different watermarks in every level.

The major drawback of DHT as well as other unitary transforms lies in their lack of space-frequency localization which is important to design robust and imperceptible watermarking. Majority of the above DHT-based watermarking, except the CHT and multiresolution-based methods, do not consider this issue while embedding watermark information. But the inherent advantage of low computation cost for DHT is comparatively increased in the latter methods. In general, DHT domain watermarking methods $[12,13]$ utilize the benefits such as low computation cost, robust watermark due to sequency effect which packs energy of the cover in the low and the middle frequency coefficients, and wide length of useful middle frequency bands with low processing noise which provides compression resilient watermarking at low quality. There is other benefit such as smaller change in image information due to watermarking, is also offered by Hadamard transform compared to DCT, DFT and DWT. To the best of our knowledge, this attribute of Hadamard transform has not been investigated in watermarking applications. This paper discusses this issue along with robust watermark design. In our method, space-frequency aspect in data hiding is considered by selecting the embedding regions based on the spatial image features. Watermark information is then casted into the transform coefficients of these regions.

The watermarking algorithm developed in this work focuses on the selection of the blocks and the coefficients to embed watermark information so that data imperceptibility is well preserved. At the same time, the watermarking scheme should also be compliant to robustness against various image processing operations including compression operation at low quality factor for both JPEG and JPEG 2000. We compare imperceptibility performance of the proposed method with $[12,13,15,16]$ as well as Cox [8] and Podilchuk [9] method. Similarly robustness performance of the proposed method is compared with the method proposed by Calagna et al. [17] as well as other Hadamard transformedbased watermarking methods [12-16]. Robustness against varieties of signal processing operations is served by embedding the same watermark signal in the low informative and the medium-informative blocks selected on the basis of spatial uncertainty and average edge information measure. The change in the values representing the Structural SIMilarity index (SSIM) [18] are used to quantify embedding distortion that matches with the perceived visual quality of the watermarked images.

The paper is organized as follows: Sections 2 and 3 describe image information measure and its use for the selection of the blocks as well as the coefficients for data embedding, respectively. Sections 4 and 5 present watermark embedding and decoding process, respectively. Performance evaluation is discussed in Section 6 while conclusion is made in Section 7.

\section{Image information for data hiding}

In this section, we highlight the usefulness of the average information (entropy) followed by suitable mathematical forms in order to select embedding regions that satisfy imperceptibility and robustness requirements of watermarking. When an image is perceived by human visual system (HVS), perceptual information is extracted not from the single pixel alone but from a group of pixels in its neighborhood. Moreover, in most of the natural images, a strong two dimensional (2D) spatial correlation exists among the neighboring pixels which results in structural information of the 
(a)

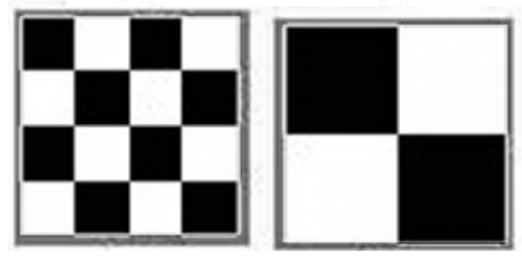

(c)

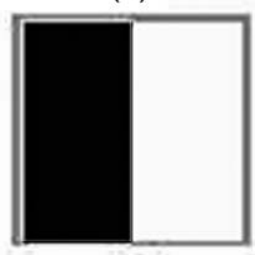

Fig. 1. Three different binary images with same entropy value.

image. One of the good measures of this spatial correlation of neighboring pixels is the average information (entropy). There are varieties of image processing applications such as segmentation, coding and compression where average information or entropy has been used extensively. Pal and Pal developed [19] new definition of entropy to achieve optimal solution of object background segmentation for gray scale image. In steganography, the security of the hidden data is measured using relative entropy distance between the cover and the watermarked data [20]. Data hiding capacity is primarily determined by the entropy of the cover $[11,21]$.

The above applications suggest that entropy of image/subimage may be used as an important image characteristics to design robust and imperceptible watermarking. If there is a noticeable change in spatial correlation of the neighboring pixels during data manipulation, image visual quality is likely to be degraded. To include the correlation among the neighboring signal points, Watson models [22] contrast masking in term of entropy. Maity et al. propose spatial domain [23] and low cost transform domain [24] watermarking using average information or entropy as image characteristics.

The widely used mathematical form to calculate entropy is shown in Eq. (1) due to Shannon, which is a global measure with respect to the image/subimage. According to Shannon's definition, the entropy of an $n$-elements set is

$H=-\sum_{i=1}^{n} p_{i} \log p_{i}$

where $p_{i}$ is the probability of occurrence of the event " $i$ " with $0 \leqslant p_{i} \leqslant 1$ and $\sum_{i=1}^{n} p_{i}=1$ [25]. The value depends solely on the probability distribution of the pixel intensities but does not consider the co-occurrence of the pixel values. That is why this measure does not always capture the actual pictorial information of the image/subimage.

Three binary images are shown in Fig. 1 where the entropy value is same for all but the pictorial information content (measure of uncertainty) is decreasing in order from Fig. 1(a) to (c). This figure reveals that subimage with a particular entropy value in a real image a may represent a smooth as well as noisy or edgy region. It is well known that edge is an important image characteristic which carries major information about any natural image. So along with the average information value, the average edge information of the image block is also considered to be important for selection of the embedding regions. It is desirable that the minimum number of edge points of the cover image should be modified during the watermark embedding. Moreover, the edges (the high spatial frequency points) are getting modified to a greater extent during lossy compression which means that the edge points are relatively unstable site for watermark embedding.

The very basic assumption of Shannon's entropy measure is to consider the signal as a long sequence of symbols. The entropy of the sequence solely depends on the relative occurrence of the symbols irrespective of their position of occurrence. It has been shown in [19] that an exponential form of entropy function can capture 2D spatial correlation of images in a better way compared to conventional Shannon's entropy. The modified form of entropy for an image block is defined in [19] as

$H=\sum_{i=1}^{n} p_{i} \exp ^{u_{i}}=\sum_{i=1}^{n} p_{i} \exp ^{1-p_{i}}$

where $u_{i}=\left(1-p_{i}\right)$ is the ignorance or uncertainty of the pixel value.

To calculate the average edge information i.e. edge entropy of the block, first the edge map is calculated using the conventional gradient operator. The edginess or the strength of edge of a pixel automatically considers the effect of neighborhood pixel values, so the measure of edge entropy of subimage is dependent on the relative occurrence of these edge strength irrespective of their position. This says that Shannon's form of entropy can be applied to calculate the average edge information of each block using the edge map.

\section{Blocks and coefficients selection for embedding}

The cover image is partitioned into $(p \times p)$ nonoverlapping block where $p=2^{n}$, and $n=1,2,3 \ldots$ If the size of the cover image is $(N \times N)$, the total $N^{2} / p^{2}$ number of blocks are obtained from the cover image, and in general $p \ll N$. The value of $p$ depends on the size of the watermark. The edge entropy or average edge information of each block is calculated from edge map using Eq. (1). The average gray information (visual) is calculated using Eq. (2). The average information due to gray level and edge entropy value of each block are added, the total values obtained are sorted in ascending order of magnitude and stored as linear chain. Fig. 2 shows the relative position of the different informative blocks. Let the size of the watermark symbol is $(M \times M)$, then two sets of $M^{2}$ blocks located at the top (shown on the left) and the bottom end (shown on the right) are termed here as the low and the high-informative blocks, respectively. The other $M^{2}$ blocks corresponding to the entropy values positioned between $\left[\left(N^{2} /\left(p^{2} * 2\right)\right]-M^{2} / 2\right.$ to $\left[\left(N^{2} /\left(p^{2} * 2\right)\right]+M^{2} / 2\right.$ in the chain is termed here as the medium-informative blocks. 


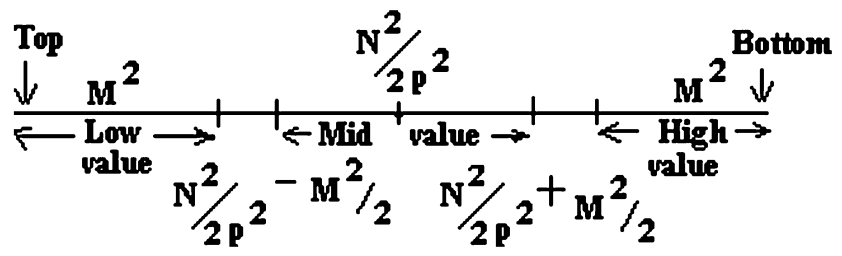

Fig. 2. Linear chain showing the relative position of different information blocks.

If watermark information is embedded in the different transform coefficients of the high-informative blocks, perceptual distortion will be less but at the same time the embedded information is fragile against lossy compression and other signal processing operations. On the other hand, watermark information embedded in the low-informative blocks may be perceived but offers high robustness against smoothing filtering. In order to make the scheme resilient against various possible signal processing operations, watermark information is embedded redundantly both in the low and the medium-informative blocks. However, appropriate transform coefficients for different blocks should be selected so that imperceptibility and robustness requirement can be satisfied properly.

It is to be noted that in bit-replacement method for watermark embedding, the pixel values remain unchanged, or increased or decreased based on the match/mismatch characteristics between the watermark bits and the bit plane of the cover. Let us see the effect of additive watermarking to the change in average gray level information and the average edge information of the image block when watermark information is embedded both in the zeroth order $\left(H_{0,0}\right)$ Hadamard coefficient and the other higher order $\left(H_{u, v}\right)$ coefficient, respectively. The inverse Hadamard transform [26] of an $(N \times N)$ (where $\left.N=2^{n}\right)$ image function $f(x, y)$ can be written as follows:

$$
\begin{aligned}
f(x, y)= & \frac{1}{N} \sum_{u=0}^{N-1} \sum_{v=0}^{N-1} H_{u, v}(-1)^{\sum_{i=0}^{n-1}\left[b_{i}(x) b_{i}(u)+b_{i}(y) b_{i}(v)\right]} \\
= & \frac{1}{N}\left[H_{0,0}(-1)^{\sum_{i=0}^{n-1}\left[b_{i}(x) b_{i}(0)+b_{i}(y) b_{i}(0)\right]}\right. \\
& +\sum_{v=1}^{N-1} H_{0, v}(-1)^{\sum_{i=0}^{n-1}\left[b_{i}(x) b_{i}(0)+b_{i}(y) b_{i}(v)\right]} \\
& +\sum_{u=1}^{N-1} H_{u, 0}(-1)^{\sum_{i=0}^{n-1}\left[b_{i}(x) b_{i}(u)+b_{i}(y) b_{i}(0 p e)\right]} \\
& \left.+\sum_{u=1}^{N-1} \sum_{v=1}^{N-1} H_{u, v}(-1)^{\sum_{i=0}^{n-1}\left[b_{i}(x) b_{i}(u)+b_{i}(y) b_{i}(v)\right]}\right]
\end{aligned}
$$

where $x, y=0,1,2 \ldots(N-1), H_{u, v}$ is the forward Hadamard transform of $f(x, y)$ for $u, v=0,1,2 \ldots(N-1)$, and $b_{k}(z)$ in the expression of kernel represents the $k$-th bit in the binary representation of $z$. The summation in the exponent is carried out in modulo- 2 arithmetic. Let the watermark information be embedded in the zeroth order coefficient $H_{0,0}$ and the embedding strength is denoted by $\Delta w$. The watermarked image denoted by $f_{1}(x, y)$, where $x, y=0,1,2 \ldots(N-1)$, can then be written as follows:

$$
\begin{aligned}
f_{1}(x, y)= & \frac{1}{N}\left[H_{0,0}+\Delta w\right](-1)^{\sum_{i=0}^{n-1}\left[b_{i}(x) b_{i}(0)+b_{i}(y) b_{i}(0)\right]} \\
& +\sum_{v=1}^{N-1} H_{0, v}(-1)^{\sum_{i=0}^{n-1}\left[b_{i}(x) b_{i}(0)+b_{i}(y) b_{i}(v)\right]} \\
& +\sum_{u=1}^{N-1} H_{u, 0}(-1)^{\sum_{i=0}^{n-1}\left[b_{i}(x) b_{i}(u)+b_{i}(y) b_{i}(0 p e)\right]} \\
& +\sum_{u=1}^{N-1} \sum_{v=1}^{N-1} H_{u, v}(-1)^{\sum_{i=0}^{n-1}\left[b_{i}(x) b_{i}(u)+b_{i}(y) b_{i}(v)\right]}
\end{aligned}
$$

The change in pixel values $\Delta f(x, y)$, due to watermark embedding, can be obtained by subtracting Eq. (3) from Eq. (4), and for $x, y=0,1,2 \ldots(N-1), \Delta f(x, y)$ can be written as follows:

$\Delta f(x, y)=(\Delta w)(-1)^{\sum_{i=0}^{n-1}\left[b_{i}(x) b_{i}(0)+b_{i}(y) b_{i}(0)\right]}=\Delta w$

In (5), $\Delta w$ is independent of $x$ and $y$ as the exponent of $(-1)$ is $\mathbf{0}$ for $b_{i}(0)=0$. This means that if $\Delta w$ amount of watermark information is added in the zeroth order Hadamard coefficient of an image block, all the pixel values within the block in the watermarked image are increased by $\Delta w$. As a result both the average information and the average edge information remain unchanged before and after watermark embedding. This fact is validated through the results obtained by performing simulations on images. Table 1 represents simulation results for the test image fishing boat shown in Fig. 3(a). The data against the first column of the table indicates the location of the respective block through the co-ordinates of the top leftmost point of the block.

On the other hand, if watermark information is embedded in the zeroth order Hadamard coefficient of the mediuminformative block, image distortion may be perceived as blocking effect due to the relatively high information content of the neighboring blocks. The relative high information content likes to mean here the information content of the block which is higher than that of low-informative neighboring block. In natural images, a particular type of block is expected to have similar type of neighboring blocks. It is logical to think that a medium-informative block is expected to have neighboring blocks with comparatively higher information content compared to the low information blocks. Watermark information embedded in the zeroth order Hadamard coefficient causes change in all pixel values of the block by the same amount and in the same direction. As a result, data embedding in a block may be perceived if the other 
Table 1. Average information and average edge information for some image blocks before and after DHT domain embedding in zeroth order coefficient.

\begin{tabular}{lllll}
\hline $\begin{array}{l}\text { Coordin. of } \\
\text { blocks }\end{array}$ & $\begin{array}{l}\text { Avg. info. before } \\
\text { embeding }\end{array}$ & $\begin{array}{l}\text { Avg. info. after } \\
\text { embeding }\end{array}$ & $\begin{array}{l}\text { Avg. edge info. } \\
\text { before embeding }\end{array}$ & $\begin{array}{l}\text { Avg. edge info. } \\
\text { after embeding }\end{array}$ \\
\hline$(0,216)$ & 2.512942 & 2.512942 & 2.667945 & 2.667945 \\
$(8,8)$ & 2.440413 & 2.440413 & 2.696327 & 2.696327 \\
$(8,160)$ & 2.552628 & 2.552628 & 3.078563 & 3.078563 \\
$(16,16)$ & 2.516566 & 2.516566 & 2.876068 & 2.876068 \\
$(16,216)$ & 2.541003 & 2.541003 & 2.844069 & 2.844069 \\
$(24,112)$ & 2.535047 & 2.535047 & 2.964950 & 2.964950 \\
\hline
\end{tabular}

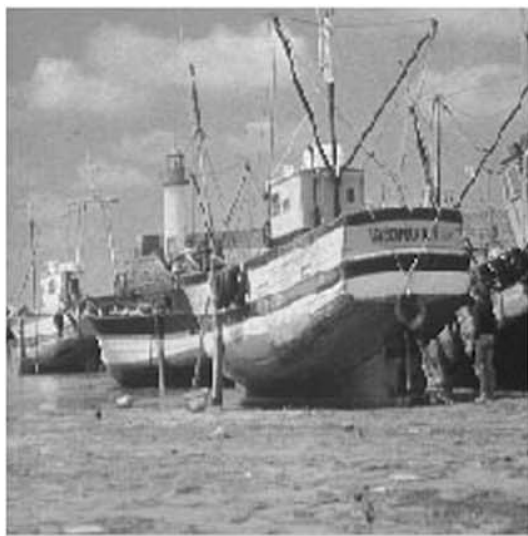

(a)

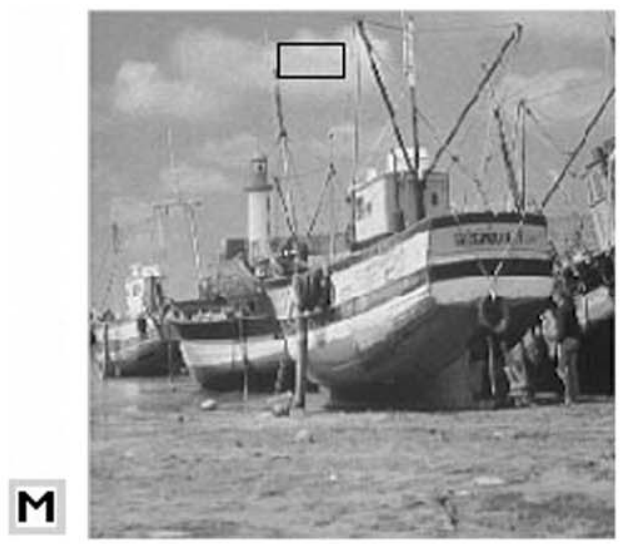

(c)

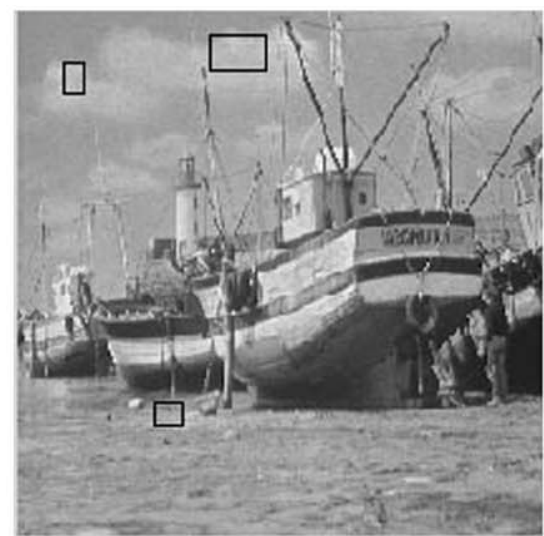

(d)

Fig. 3. (a) Test image boat, (b) watermark image, (c) watermarked image using Hadamard transform, (d) watermarked image using DCT transform.

neighboring blocks is not selected as embedding region. To solve the problem, watermark information is embedded in the higher order coefficient of the block. It can be shown mathematically that for additive watermarking in any higher order Hadamard coefficient, half of the pixel values of a block are increased and the values for the remaining pixels are decreased. However, the amount of change is the same in both the cases. We rewrite Eq. (4) for data embedding in $H_{j, k}$ where $j, k \neq 0$ :

$$
\begin{aligned}
f_{1}(x, y)= & {\left[H_{j, k}+\Delta w\right](-1)^{\sum_{i=0}^{n-1}\left[b_{i}(x) b_{i}(j)+b_{i}(y) b_{i}(k)\right]} } \\
& +\sum_{u=0, u \neq j}^{N-1} \sum_{v=0, v \neq k}^{N-1} H_{u, v}(-1)^{\sum_{i=0}^{n-1}\left[b_{i}(x) b_{i}(u)+b_{i}(y) b_{i}(v)\right]}
\end{aligned}
$$

where $x, y=0,1,2 \ldots(N-1)$. The change in the pixel values, due to watermark embedding, can be obtained by subtracting Eq. (3) from (6) and is expressed as follows:

$$
\begin{aligned}
\Delta f(x, y) & =\Delta w(-1)^{\sum_{i=0}^{n-1}\left[b_{i}(x) b_{i}(j)+b_{i}(y) b_{i}(k)\right]} \\
& = \pm \Delta w
\end{aligned}
$$

where the exponent of $(-1)$ is $\mathbf{0}$ for half of the cases and $\mathbf{1}$ for the remaining cases according to the property of Hadamard kernel. So the pixel values are increased or decreased by $\Delta w$, respectively, and the above relation is true for any $u=l, v=k$ where $l, k \neq 0$. If watermark information is embedded in the coefficient $u=l, v=k$ where $l, k \neq 0$ for other transformation, say DCT, the change in pixel values can be written similar to Eq. (7) as follows:

$$
\begin{aligned}
\Delta f(x, y) & =f_{1}(x, y)-f(x, y) \\
& =\Delta w \cos \left[\frac{(2 x+1) l \pi}{2 N}\right] \cos \left[\frac{(2 y+1) k \pi}{2 N}\right]
\end{aligned}
$$

Eq. (8) shows that the amount of changes are different for different pixels and the value of change also depends on the choice of the particular coefficient to be used for embedding i.e. $u$ and $v$ values. If we calculate the average information or entropy of the change in pixel values, Hadamard domain embedding causes small change (as change in the pixel values within a block are of two types $+\Delta w_{i}$ or $-\Delta w_{i}$ leading to less amount of uncertainty) compared to DCT domain embedding (as change in pixel values are of different possible types leading to more uncertainty). The result is also true for other popular transforms such as DFT, Fourier-Mellin, and wavelet, etc. So the results in Eqs. (7) and (8) when put into Eqs. (1) or (2) can be summarized as follows:

(1) Image information is changed by less amount in case of Hadamard domain embedding compared to other 
popular transform domain embedding as in the latter cases different pixel values are changed by different amount due to the multivalued kernels.

(2) For a given embedding distortion, bit plane for data hiding can be selected easily in case of Hadamard transform compared to other transformations.

It is to be noted that the results obtained in (5), (7) and (8) are valid for any value of $\Delta w$ and the value should not be fixed for the entire image i.e. for all the subimages chosen for watermarking. The value $\Delta w$ must be adaptive based on the local characteristics of image. For example, if watermark information is embedded in the smooth region, the embedding strength i.e. $\Delta w$ value would be small but the same may be chosen high if the embedding region corresponds to texture or edge. One simple way of implementation is to consider the mean gray values of the image blocks. Watermark information is embedded adaptively by choosing the different bit planes of these mean gray values. The other advantages of the DHT domain embedding methods are shorter processing time due to real valued kernel and the usage of the same algorithm for the forward and the inverse transformation (identical and orthogonal nature of kernels [26], and ease of hardware implementation due to binary valued kernel) [27-29].

\section{Proposed watermarking technique}

We propose here the watermarking method for the cover image which is a gray-scale image of size $(N \times N)$ and the watermark is a binary image of size $(M \times M)$. However, the method can also be extended to color image. The color image is first transformed from RGB into luminance/chrominance color space such as $Y C_{b} C_{r}$ where the symbols R, G, B, $\mathrm{Y}, C_{b}$ and $C_{r}$ denote the red, the green, the blue, the luminance, the chrominance-blue and the chrominance-red, respectively. The image is then partitioned into different nonoverlapping blocks in $\mathrm{Y}$ channel. The blocks for watermark embedding are selected based on the average information and the average edge information as discussed in Section 3. Watermark information is then embedded according to the different steps as stated below. The computational cost of the watermarking method for the color image is exactly the same as required for gray scale image except the extra computational cost of transformation from RGB to $Y C_{b} C_{r}$. However, as mentioned earlier, we propose here the method for the gray scale image and the different steps for watermark embedding are described as follows:

Step I: Spatial dispersion of watermark image. The binary watermark image is spatially dispersed $\left(L_{1}\right)$ using a $M^{2}$-bit cryptic key $\left(k_{1}\right)$ generated by a linear feedback shift register (LFSR) [30].

Step II: Watermark embedding in low-informative blocks and formation of secret image $S_{1}$. Fast Hadamard transformation is applied on each low- and medium-informative block of size $(p \times p)$ pixels for the cover image. Let $H_{u, v, b}$ be the $(u, v)$-th coefficients of the block $b$, where $H_{0,0, b}$ is the zeroth order Hadamard coefficient of the same. The integer part of this coefficient, i.e. $\left\lfloor H_{0,0, b}\right\rfloor$ for each block is denoted by $\widetilde{H}_{0,0, b}$. Since the cover image is 255 level gray tone image, the bit plane representation of $\widetilde{H}_{0,0, b}$ needs at most eight bits and is expressed by $a_{7} a_{6} a_{5} a_{4} a_{3} a_{2} a_{1} a_{0}$. A suitable lower order bit (preferably any one of $a_{3} a_{2} a_{1}$ ) of $\widetilde{H}_{0,0, b}$, is replaced by one binary pixel from spatially dispersed watermark symbol $L_{1}$. The choice of bit plane depends on the average brightness and the contrast of the pixel values in a block. The third or fourth LSB (least significant bit) plane is selected preferably in order to make a good compromise between the robustness and imperceptibility. Watermark embedding in the higher order bit plane may cause greater visual distortion but robustness performance is also improved. Moreover, the selection of higher bit plane offers flexibility to implement adaptive negative modulation (as described later in step IV) in order to achieve higher robustness against compression operation. The higher bit plane may be chosen for data embedding provided the mean gray value of the block is either less than $T_{1}$ or greater than $T_{2}$, where $T_{1}$ and $T_{2}$ are certain prespecified threshold values with $T_{1}$ should preferably be close to ' 0 ' (minimum) and $T_{2}$ close to '255' (maximum). We have done experiment over a large number of images. As a rule of thumb, for mean gray value of the block less than 50, 100 and 150, LSB planes fourth, third and second one can be selected for embedding, respectively. The fractional part of $H_{0,0, b}$ is now appended with the modified $\widetilde{H}_{0,0, b}$ value for each such block.

A three-level intermediate image map $S_{1}$ of size $(N / p \times$ $N / p$ ) is constructed where each block of the cover image is mapped as a point in the intermediate image. A 2-bit code is required to represent the three levels, the low-, mediuminformative, and the remaining blocks. This image is used to identify the type of the blocks in the possibly distorted watermarked image during extraction of watermark.

Step III: Watermark embedding in medium-informative blocks and formation of secret image $S_{2}$. The same watermark bits are also embedded in the medium-informative image blocks. For each block, the higher order coefficient with the highest magnitude (ignoring its sign) i.e. $\max \left(H_{u, v, b}\right) \neq H_{0,0, b}$ is selected. The basic assumption of embedding watermark information in the highest valued non-zero order Hadamard coefficient is that the embedded coefficient is most likely to survive after quantization operation of lossy compression. The integer part of the coefficient is denoted by $\bar{H}_{u, v, b}$ and a suitable lower order bit of $\bar{H}_{u, v, b}$ is replaced by one bit of the spatially dispersed watermark symbol $L_{1}$. The bit plane for watermark embedding is selected in the similar method as described in step II. The fractional part of $\max \left(H_{u, v, b}\right)$ is now appended with the modified $\bar{H}_{u, v, b}$ value for each such block.

The secret image $S_{2}$ of size $(M \times M)$ is formed, where each pixel value of the image represents the positional information of the desired highest Hadamard coefficient within 
the medium-informative block. The secret image $S_{2}$ is a $p^{2}$ level gray tone image and the size of the image is $(M \times M)$ which is equal to the size of the binary watermark image. The first pixel value of this image represents the positional information of the coefficient in first medium-informative block of secret image $S_{1}$, the second pixel value of secret image $S_{2}$ for the coefficient of second medium-informative block of secret image $S_{1}$ and so on.

Step IV: Robustness against compression using adaptive negative modulation. To increase the robustness efficiency of the proposed scheme against lossy compression, an attack adaptive negative modulation is used (after watermark embedding) only for medium-informative blocks. The negative modulation refers to the change in the value of the embedded coefficients in the direction opposite to that of the expected coefficient values obtained after lossy compression. This adaptive modulation enables to retain the watermark information after a certain extent of quantization operation during compression. The implementation of negative modulation is accomplished assuming that the watermarked image is going to undergo a particular type of compression operation, say JPEG i.e. the particular compression operation is assumed to be known a priori. Although this negative modulation improves robustness against such compression operation significantly, however, its performance against other type of compression, say JPEG 2000, is also increased as both types of compression operations are identical in nature. The benefit of compression resiliency is achieved at the cost of computation for implementation of compression operation. However, computation cost for further manipulation needed to complete the negative modulation is not high. The implementation of negative modulation process is described as follows.

Let us assume that the desired Hadamard coefficient $\bar{H}_{u, v, b}$ of a medium-informative block after watermark embedding becomes $\bar{H}_{u, v, b}^{1}$. It is also assumed that the binary watermark pixel " 1 " (say) is embedded in the third LSB i.e. in $a_{2}$ of the bit plane representation of $\bar{H}_{u, v, b}$ and three LSBs of $\bar{H}_{u, v, b}^{1}$ i.e. $a_{2} a_{1} a_{0}$ now become 1,1 , and 0 assuming $a_{1} a_{0}$ of $\bar{H}_{u, v, b}$ as 00 . After lossy compression the coefficient is expected to change to the value $\bar{H}_{u, v, b}^{2}$ (say). Three possible manipulations are made as follows.

(1) If $\bar{H}_{u, v, b}^{2}>\bar{H}_{u, v, b}^{1}$ then two LSBs of $\bar{H}_{u, v, b}^{1}$ i.e. $a_{1} a_{0}$ are forced to " 0 " so that three LSBs i.e. $a_{2} a_{1} a_{0}$ of $\bar{H}_{u, v, b}^{1}$ now become 100 .

(2) If $\bar{H}_{u, v, b}^{2}<\bar{H}_{u, v, b}^{1}$ then two LSBs of $\bar{H}_{u, v, b}^{1}$ i.e. $a_{1} a_{0}$ are forced to " 1 " so that three LSBs i.e. $a_{2} a_{1} a_{0}$ of $\bar{H}_{u, v, b}^{1}$ now become 111 .

(3) If $\bar{H}_{u, v, b}^{2}=\bar{H}_{u, v, b}^{1}$ no bit manipulation is made.

The negative modulation helps to improve the visual quality of the watermark symbol extracted from the compressed watermarked image at the cost of marginal visual degradation of the watermarked image. Watermark information is embedded in LSB plane of the coefficient and negative modulation is implemented by manipulating other bit planes. Simulation results done over large number of images show that this visual distortion is of the order of $(\sim 1.0 \mathrm{~dB})$ and could hardly be perceived by HVS. This negative modulation is termed as "compression like attack adaptive negative modulation".

Step V: Formation of watermarked image. Block-based fast inverse Hadamard transformation is applied next on the set of blocks obtained after watermark embedding. These sets of blocks and non-watermarked blocks of the cover image are then placed in the proper positions of the cover image, and the watermarked image is obtained.

\section{Extraction of watermark}

The extraction of watermark symbol requires the cryptic key $k_{1}$, the secret images $S_{1}$ and $S_{2}$. It is already shown in Section 3 that additive watermarking in zeroth order Hadamard coefficient does not change average edge information as well as average gray information (visual) of a block. It is also shown that watermark embedding in the higher order coefficients except the zeroth order, causes the change in average edge information as well as average gray information comparatively much smaller in case of DHT domain embedding than that of DCT domain embedding.

It is expected that the average information and the average edge information of the watermarked image blocks will change after various common or deliberate signal processing operations. This may be treated as de-synchronization in watermark extraction process. So it is important not only to identify the blocks in the watermarked images after attacks that contain watermarks but also to distinguish them as the low- and the medium-informative blocks. The watermarked image blocks after signal processing operations can be identified using the secret images $S_{1}$ and $S_{2}$. Secret image $S_{1}$ provides information about the low- and the medium-informative blocks while the secret image $S_{2}$ provides the positions of the highest coefficients in each medium-informative blocks. This information is used for watermark extraction from the watermarked image under various external attacks and solves the de-synchronization problem in watermark extraction process.

Step I: Selection of watermarked image blocks. The distorted watermarked image is now partitioned into nonoverlapping block of size $(p \times p)$ pixels. The positions of the low- and the medium-informative blocks of the distorted watermarked image are identified using the secret image $S_{1}$.

Step II: Watermark extraction from low- and mediuminformative blocks. Block-based Hadamard transform is then applied for all the low- and the medium-informative blocks. From each low-informative block, one watermark pixel is extracted from the proper bit after the bit plane representation 
of the integer part of the zeroth order Hadamard coefficient. Similarly, the watermark symbol can also be extracted from the medium-informative blocks. One watermark pixel is extracted from the proper bit position of each of the desired Hadamard coefficient i.e. the largest Hadamard coefficient. The bit planes for extraction of watermark information can be selected based on the thumb rule as discussed in step II of Section 4

Step III: Spatial dispersion of the extracted watermark. The spatially dispersed watermark symbol thus obtained from each region is rearranged using the key $k_{1}$. The watermark symbols, extracted from the two different regions, preserve different visual recognizability based on the nature and depth of external attacks/signal processing operations.

A quantitative estimation of the extracted image $W^{\prime}(x, y)$ quality may be expressed as normalized cross-correlation (NCC) [31] where

$N C C=\frac{\sum_{x} \sum_{y} W(x, y) W^{\prime}(x, y)}{\sum_{x} \sum_{y}[W(x, y)]^{2}}$

which is the cross-correlation normalized by the watermark energy to give the maximum value of NCC as unity. The symbol $W(x, y)$ indicates the original binary watermark image.

\section{Performance evaluation}

The proposed watermark embedding methodology is based on the combination of the selection of the embedding regions using image information measure and adaptive negative modulation to enhance resiliency against lossy compression. The watermark is a binary image of size $(16 \times 16)$ and the cover image is a gray-scale image of size $(256 \times 256), 8$ bits/pixel. The proposed algorithm requires approximately 6 seconds for watermark embedding and approximately 4 seconds for extraction in DHT domain while 9 seconds for embedding and 7 seconds for decoding in DCT domain using Visual $\mathrm{C} / \mathrm{C}++$ platform running on a Pentium III $400 \mathrm{MHz}$ PC system. The runtime breakdown process of the proposed watermark embedding method shows that spatial dispersion of watermark, region selection based on average information and edge information of blocks followed by sorting process takes approximately 3 seconds, DHT decomposition and watermark embedding in two regions consumes 1 second, adaptive negative modulation and the formation of the watermarked image through inverse DHT takes 2 seconds. Similarly, the runtime breakdown of watermark decoding process shows that selection of watermark embedding blocks (without the use of $S_{1}$ and $S_{2}$ ) from the cover image takes 3 seconds and the watermark extraction process through DHT decomposition takes 1 second. It is to be noted that if we use secret images $S_{1}$ and $S_{2}$ to find the embedded regions, watermark extraction process takes very less time.
We study the performance of the proposed watermarking method over large number of benchmark images [32,33]. Fig. 3(a) (fishing boat) shows an original test image and Fig. 3(c) and (d) show the watermarked images for DHT and DCT domain embedding, respectively, using logo/hidden symbol ' $M$ ' of Fig. 3(b). The rectangular boxes shown in Fig. 3(c) and (d) indicate the areas where the visually distinguishable distortions occur. The embedding regions are same in both cases determined on the basis of proposed image information measure. Visual distortion shown in Fig. 3(c) is very low and could hardly be perceived while there are noticeable distortions in few places in Fig. 3(d), possibly due to the contribution of entropy masking resulted from higher change in entropy due to DCT domain embedding. This subjective visual qualities of the watermarked images are also supported by the objective measures. The PSNR and MSSIM values between the watermarked image and the original image are $39.62 \mathrm{~dB}$ and 0.9872 for DHT domain embedding and $35.24 \mathrm{~dB}$ and 0.9546 for DCT domain embedding.

We compare the perceptual transparency results of our algorithm with several other DHT domain watermarking methods $[12,13,15,16]$ as well as other two popular watermarking methods [9] (Podilchuck algorithm of DCT domain implementation) and [8] (Cox algorithm). The simulation results are shown in Table 2 for few cover images, although the experiment has been carried out over large number of benchmark images [32,33]. Fig. 4(a)-(d) show four other test images, the results of which are shown in Table 2. Simulation results show that the proposed method always offers better data imperceptibility compared to other watermarking methods. When boat image is used as test image, PSNR values are $34.34 \mathrm{~dB}$ for [15], $34.23 \mathrm{~dB}$ for [16], $35.76 \mathrm{~dB}$ for [13], $34.32 \mathrm{~dB}$ for [12], $31.23 \mathrm{~dB}$ for [8], $35.45 \mathrm{~dB}$ for [9] and $39.62 \mathrm{~dB}$ for the proposed scheme. Similar objective quality differences $(\sim 39.5 \mathrm{~dB})$ are also found when the values are averaged for large number of test images using the proposed method. Moreover, alteration in structural information due to data embedding occurs least for the proposed method compared to the other methods. While comparing the perceptual invisibility of the hidden data, differences in visual quality become apparent for the proposed method with respect to other DHT domain watermarking methods as the latter methods do not consider image characteristics while selecting the embedding regions. Visual quality of the watermarked images are relatively good for $[9,13]$. This is because the work in [13] uses edge and texture characteristics to calculate embedding strength while [9] uses characteristics of HVS. The selection of DHT as signal decomposition also offers benefits of low loss in image information due to watermark embedding.

Fig. 5 shows graphically the average absolute difference in edge entropy values for the medium-informative blocks both in DCT and DHT domain embedding. The horizontal axis of Fig. 5 represents those 256 blocks appeared as mediuminformative blocks in the ascending order arrangement. We 
Table 2. Comparison of perceptual transparency for Falk [15], Kuon [16], Ho [13], Li [12], Cox [8] and Podilchuk [9] algorithms.

\begin{tabular}{|c|c|c|c|c|c|c|c|}
\hline $\begin{array}{l}\text { Test } \\
\text { image }\end{array}$ & $\begin{array}{l}\text { PSNR MSSIM } \\
\text { value, Prop. }\end{array}$ & $\begin{array}{l}\text { PSNR MSSIM } \\
\text { value, Falk [15] }\end{array}$ & $\begin{array}{l}\text { PSNR MSSIM } \\
\text { value, Kuon [16] }\end{array}$ & $\begin{array}{l}\text { PSNR MSSIM } \\
\text { value, Ho [13] }\end{array}$ & $\begin{array}{l}\text { PSNR MSSIM } \\
\text { value, } \mathrm{Li}[12]\end{array}$ & $\begin{array}{l}\text { PSNR MSSIM } \\
\text { value, Cox [8] }\end{array}$ & $\begin{array}{l}\text { PSNR MSSIM } \\
\text { value, Podl. [9] }\end{array}$ \\
\hline Boat & $\begin{array}{l}39.62 \\
0.9766\end{array}$ & $\begin{array}{l}34.34 \\
0.9532\end{array}$ & $\begin{array}{l}34.23 \\
0.9424\end{array}$ & $\begin{array}{l}35.76 \\
0.9732\end{array}$ & $\begin{array}{l}34.32 \\
0.9441\end{array}$ & $\begin{array}{l}31.23 \\
0.9242\end{array}$ & $\begin{array}{l}35.45 \\
0.9441\end{array}$ \\
\hline Bear & $\begin{array}{l}38.49 \\
0.9887\end{array}$ & $\begin{array}{l}35.67 \\
0.9668\end{array}$ & $\begin{array}{l}35.78 \\
0.9664\end{array}$ & $\begin{array}{l}37.89 \\
0.9786\end{array}$ & $\begin{array}{l}34.32 \\
0.9784\end{array}$ & $\begin{array}{l}31.23 \\
0.9264\end{array}$ & $\begin{array}{l}35.45 \\
0.9324\end{array}$ \\
\hline Opera & $\begin{array}{l}39.56 \\
0.9731\end{array}$ & $\begin{array}{l}34.45 \\
0.9378\end{array}$ & $\begin{array}{l}32.12 \\
0.9326\end{array}$ & $\begin{array}{l}36.24 \\
0.9521\end{array}$ & $\begin{array}{l}36.98 \\
0.9667\end{array}$ & $\begin{array}{l}31.12 \\
0.9267\end{array}$ & $\begin{array}{l}36.98 \\
0.9452\end{array}$ \\
\hline Lena & $\begin{array}{l}40.02 \\
0.9812\end{array}$ & $\begin{array}{l}36.12 \\
0.9421\end{array}$ & $\begin{array}{l}33.34 \\
0.9347\end{array}$ & $\begin{array}{l}37.45 \\
0.9613\end{array}$ & $\begin{array}{l}36.23 \\
0.9456\end{array}$ & $\begin{array}{l}30.34 \\
0.9147\end{array}$ & $\begin{array}{l}36.23 \\
0.9456\end{array}$ \\
\hline Pill & $\begin{array}{c}40.12 \\
0.9698\end{array}$ & $\begin{array}{l}36.87 \\
0.9610\end{array}$ & $\begin{array}{l}33.05 \\
0.9373\end{array}$ & $\begin{array}{l}37.21 \\
0.9596\end{array}$ & $\begin{array}{l}36.23 \\
0.9373\end{array}$ & $\begin{array}{l}31.05 \\
0.9273\end{array}$ & $\begin{array}{l}37.23 \\
0.9523\end{array}$ \\
\hline
\end{tabular}

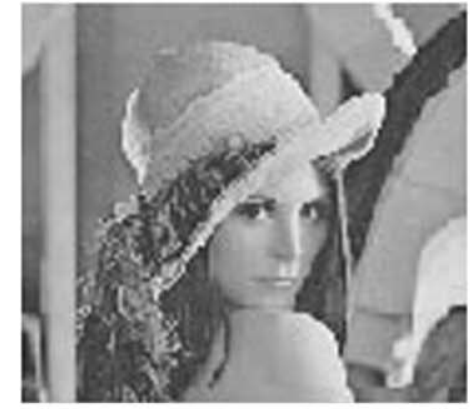

(a)

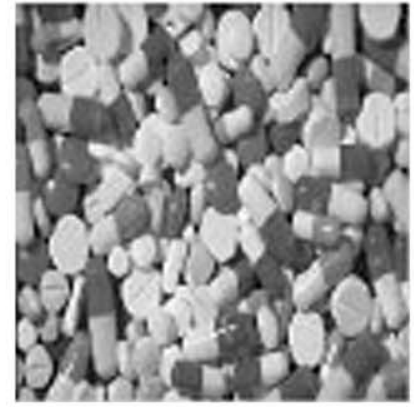

(b)

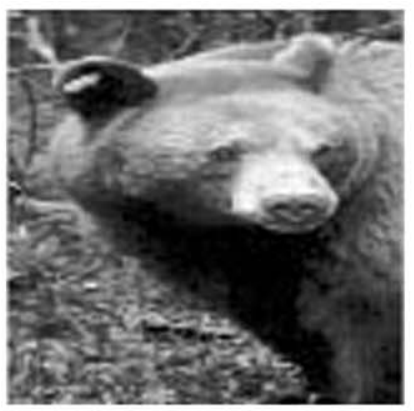

(c)

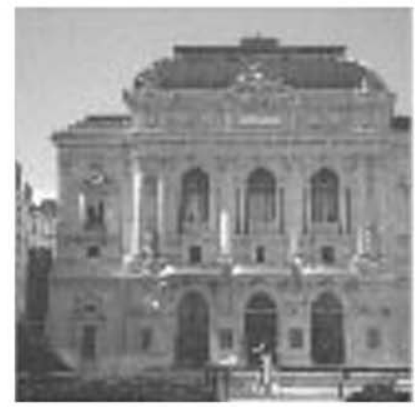

(d)

Fig. 4. Test image, (a) Lena, (b)pills, (c) bear, (d) opera.

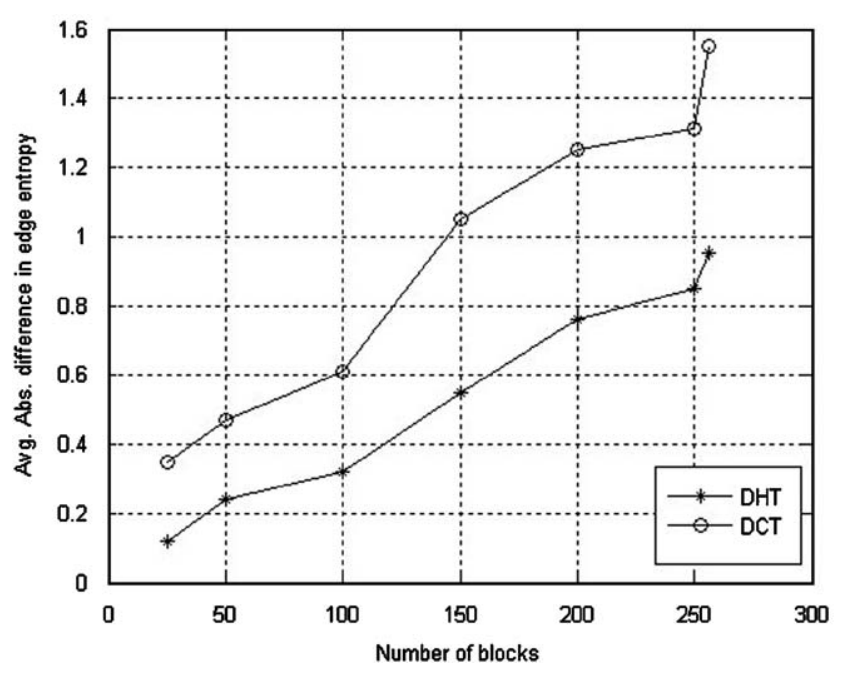

Fig. 5. Average absolute difference in edge entropy of the medium-informative blocks due to embedding.

test the effect of watermark embedding over large number of benchmark images and the numerical values shown in the vertical axis of Fig. 5 are computed by averaging over all these test images. It is clear from Fig. 5 that average absolute difference in edge entropy for the blocks due to DHT domain embedding are lower compared to the same for DCT domain embedding. This result is well supported by the result in Eq. (8) when incorporated in Eq. (1). This fact is also supported by the results shown in Table 3 for the test image fishing boat.

Robustness of the proposed watermarking method has been tested against various common image degradations as well as attacks available in checkmark package [34]. Typical image processing operations performed over the watermarked images include mean, median and Wiener filtering, image cropping, rescaling, sharpening, noise addition, histogram equalization, change in aspect ratio, image rotation and low quality JPEG and JPEG 2000 compression. Although it is difficult to correlate the recognizability of the watermark pattern with NCC values, the experiment results suggest that NCC value $(\sim 0.65)$ can be considered as threshold of recognizability for the watermark image shown in Fig. 3(b). Robustness performance of the proposed method varies with or without the usage of secret images $S_{1}$ and $S_{2}$ during watermark decoding. We report here robustness performance using secret images $S_{1}$ and $S_{2}$ 
Table 3. Change in average information and average edge information for some image blocks before and after DHT and DCT domain embedding in higher order coefficient.

\begin{tabular}{lllll}
\hline $\begin{array}{l}\text { Coordinates } \\
\text { blocks }\end{array}$ & $\begin{array}{l}\text { Avg. info. in } \\
\text { DHT, Prop. }\end{array}$ & $\begin{array}{l}\text { Avg. info. in } \\
\text { DHT, Li [12] }\end{array}$ & $\begin{array}{l}\text { Avg. edge info. in } \\
\text { DCT, Cox [8] }\end{array}$ & $\begin{array}{l}\text { Edge info. in } \\
\text { DCT, Podilchuk [9] }\end{array}$ \\
\hline$(0,56)$ & 0.00013 & 0.02236 & 0.00087 & 0.17847 \\
$(0,64)$ & 0.00014 & 0.13466 & 0.00038 & 0.48898 \\
$(8,176)$ & 0.00113 & 0.27922 & 0.00013 & 0.22051 \\
$(8,184)$ & 0.00102 & 0.30776 & 0.01083 & 0.55026 \\
\hline
\end{tabular}

and the performance is little inferior when the secret images are not used.

It is to be noted that we embed a visually recognizable binary watermark image rather than embedding a single bit watermark. Thus the extracted/decoded watermark for the proposed method is recognized by the visual quality unlike the correlation-based detection principle of single bit watermark where a particular threshold value indicates whether the watermarked image contains a particular watermark or not. Although the embedding capacity of the proposed method is suffered as the same watermark symbol is embedded in two different types of blocks redundantly in order to achieve robustness against diverse types of signal processing operations, still the capacity is reasonably good. We report here robustness performance for the proposed method with a embedding capacity of 256 bits for a cover image of size $(256 \times 256)$ by embedding a single watermark bit in a block of size $(8 \times 8)$. This embedding capacity can further be increased using the unwatermarked regions between the low- and the medium-information blocks and also by reducing the size of the embedding block. We can embed more 128 watermark bits using the unwatermarked regions between the low- and the medium-informative blocks and PSNR and MSSIM values for the test image fishing boat become $38.23 \mathrm{~dB}$ and 0.9792 , respectively. The payload capacity can be increased 4 times i.e. 1024 watermark bits can be embedded redundantly in the same cover image by reducing the size of embedding block from $(8 \times 8)$ to $(4 \times 4)$. However, imperceptibility of hidden data is affected now and PSNR and MSSIM values go down to $36.53 \mathrm{~dB}$ and 0.9634 , respectively, for the test image fishing boat. This loss in visual quality is also accompanied by the decrease in robustness performance of the hidden data i.e. degradation in visual recognizability of the extracted watermark under various signal processing operations. So the further payload improvement depends on the characteristics and the content of the cover image and with a reasonably good trade-off between visual distortion and robustness.

Fig. 6(a)-(t) show the watermarked images after various signal processing operations along with the corresponding extracted watermarks. In all cases, the extracted watermarks are visually recognizable and thus indicates that the proposed method is robust against such types of signal manipulation. Experimental results show that data embedding in the mid-informative blocks offer robustness against dynamic range change of the gray values, noise addition, image sharpening, lossy compression operations while data embedding in the low-informative blocks are resilient against various types of smoothing filtering operations. The detection overhead of the present scheme is lower compared to that of [13] since our watermark extraction process needs only the watermark embedding positions but the latter method requires the watermark embedding positions as well as the embedding strength factor corresponding to each embedding position.

Robustness of the proposed algorithm against different attacks available in the checkmark package [34] is shown in Table 4 . While comparing the robustness performance for possible attacks of checkmark with $[8,12,13,17]$, simulation results show that the proposed watermarking method offers higher degree of robustness compared to the other methods. Simulation results also show that when the extracted watermarks for the present method maintain visual recognizability even after higher degree of signal distortions, watermarking method in [17] fails to identify the proper watermark due to the low correlation values. Poor robustness of the latter method is due to the significant residual correlation between the watermark and the distortions that largely affects the selection of threshold value.

Fig. 7(a)-(d) show the efficiency of the adaptive negative modulation against lossy compression operations. PSNR values for the compressed watermark images (quality factor 20) shown in Fig. 7(a) (without negative modulation) and in Fig. 7(c) (with negative modulation) are 18.25 and $17.30 \mathrm{~dB}$, respectively. NCC values for the extracted watermarks are 0.71 and 0.88 , respectively. Results indicate that although negative modulation causes visual distortions of the watermarked images marginally but at the same time robustness performance of the embedded watermark is significantly increased.

Fig. 8(a)-(b) show graphically the robustness performance of the proposed algorithm against JPEG and JPEG 2000 compression operations using Hadamard, and DCT transform. In the low-informative block, due to embedding in zeroth order DHT coefficients, all the pixel values are changed by same amount and in the same direction. 


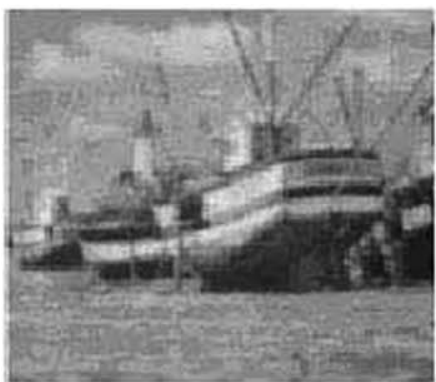

(a)

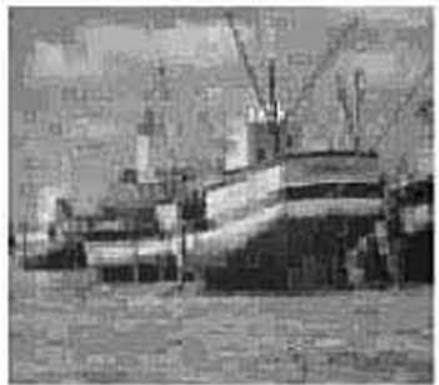

(g)

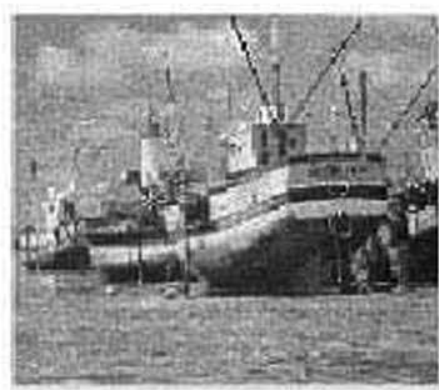

(m)

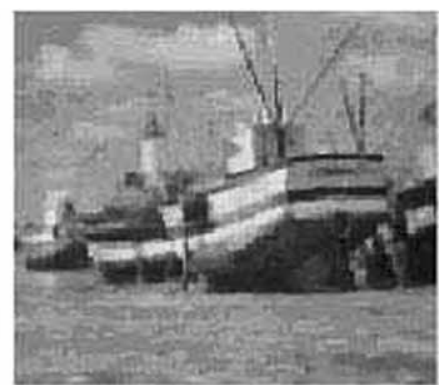

(c)

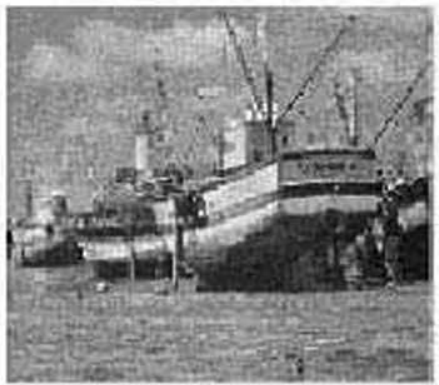

(i)

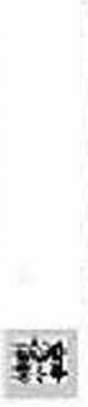

(n)

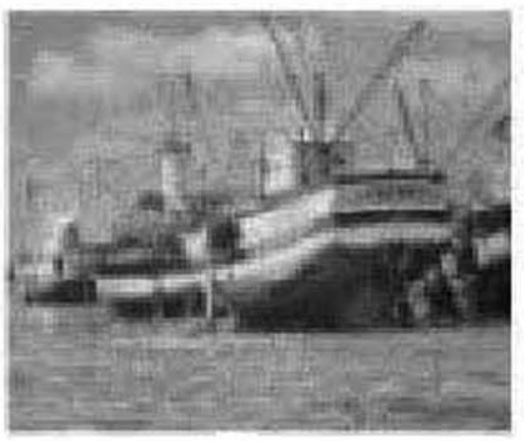

(o)

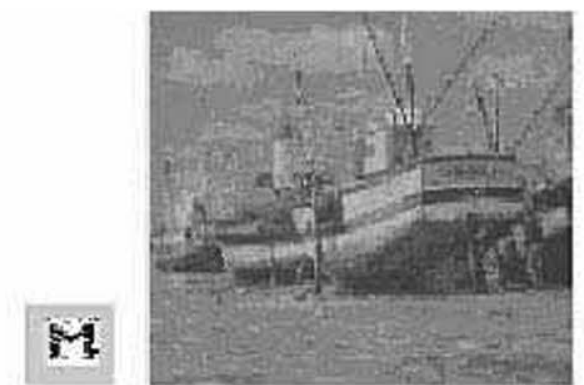

(e)

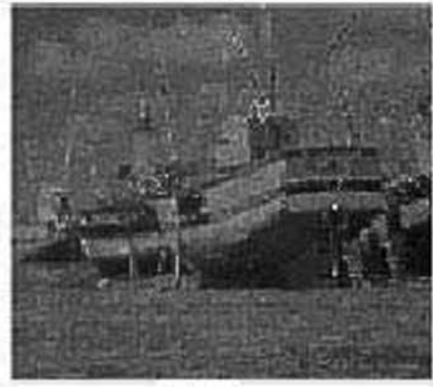

(k)

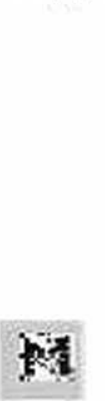

(j)

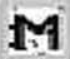

(f)

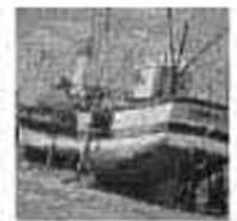

(q)

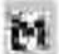

(r)

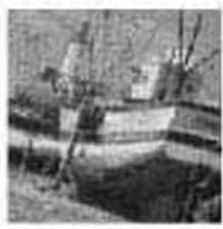

(s)

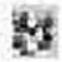

(t)

Fig. 6. (a) Watermarked image after mean filtering, (b) extracted watermark from (a), (c) watermarked image after median filtering, (d) extracted watermark from (c), (e) watermarked image after change in dynamic range, (f) extracted watermark from (e), (g) watermarked image after JPEG compression, (h) extracted watermark from (g), (i) watermarked image after LSB(s) manipulation, (j) extracted watermark from (i), (k) watermarked image after image sharpening, (l) extracted watermark from $(\mathrm{k}),(\mathrm{m})$ watermarked image after noise addition, $(\mathrm{n})$ extracted watermark from $(\mathrm{m}),(\mathrm{o})$ watermarked images after change in aspect ratio $(X=1.2$ and $Y=0.8)$, (p) extracted watermark from (o), (q) watermarked images after 10 degree rotation, (r) extracted watermark from (q), (s) watermarked image after $15^{\circ}$ rotation, (t) extracted watermark from (s).

Embedded watermark information are lost more after quantization operation of lossy compression. The loss of embedded watermark information is comparatively less in case of data embedding in the mid-informative block as watermark information are embedded in the highest coefficient other than the mean coefficient. Experiment results support that the selection of Hadamard transform as signal decomposition tool shows better performance at low quality compression compared to DCT, when both type of compression operations are taken into consideration. The graphical results also show that robustness performance is further improved using adaptive negative modulation during data embedding in the mid-informative blocks. We implement negative modulation for JPEG compression and as a matter of fact better performance improvement is achieved for JPEG compression compared to JPEG 2000. However, Fig. 8(b) shows that adaptive negative modulation based on JPEG also improves robustness performance against JPEG 2000 compression operation. Similar results are also found when Hadamard domain implementation is compared with wavelet domain implementation of the algorithm.

We also compare the robustness performance of the proposed method against JPEG and JPEG 2000 compression 
Table 4. Test results of checkmark package for the prop., Cox, Ho, Li, Calagna methods.

\begin{tabular}{|c|c|c|c|c|c|}
\hline Name of attacks & $\begin{array}{l}\text { NCC value, } \\
\text { Prop. }\end{array}$ & $\begin{array}{l}\text { NCC value, } \\
\text { Ho [13] }\end{array}$ & $\begin{array}{l}\text { NCC value, } \\
\mathrm{Li}[12]\end{array}$ & $\begin{array}{l}\text { NCC value, } \\
\text { Cala. [17] }\end{array}$ & $\begin{array}{l}\text { NCC value, } \\
\text { Cox [8] }\end{array}$ \\
\hline Wiener filtering & 0.78 & 0.71 & 0.62 & 0.72 & 0.70 \\
\hline dpr & 0.72 & 0.66 & 0.62 & 0.68 & 0.62 \\
\hline dprcorr & 0.76 & 0.64 & 0.61 & 0.69 & 0.67 \\
\hline Midpoint & 0.78 & 0.70 & 0.60 & 0.74 & 0.71 \\
\hline Threshold & 0.79 & 0.71 & 0.70 & 0.76 & 0.72 \\
\hline Hard threshold & 0.79 & 0.73 & 0.70 & 0.76 & 0.70 \\
\hline Soft threshold & 0.74 & 0.68 & 0.66 & 0.71 & 0.67 \\
\hline Sample downup & 0.82 & 0.73 & 0.70 & 0.74 & 0.67 \\
\hline dither & 0.47 & 0.51 & 0.42 & 0.56 & 0.46 \\
\hline Trimed mean & 0.79 & 0.61 & 0.67 & 0.73 & 0.67 \\
\hline Copy-collage & 0.43 & 0.52 & 0.56 & 0.49 & 0.48 \\
\hline Projective & 0.74 & 0.69 & 0.64 & 0.70 & 0.72 \\
\hline Ratio & 0.76 & 0.70 & 0.71 & 0.72 & 0.69 \\
\hline rowcol & 0.76 & 0.68 & 0.70 & 0.69 & 0.68 \\
\hline Shearing & 0.69 & 0.58 & 0.56 & 0.60 & 0.59 \\
\hline Warping & 0.67 & 0.59 & 0.58 & 0.61 & 0.62 \\
\hline
\end{tabular}

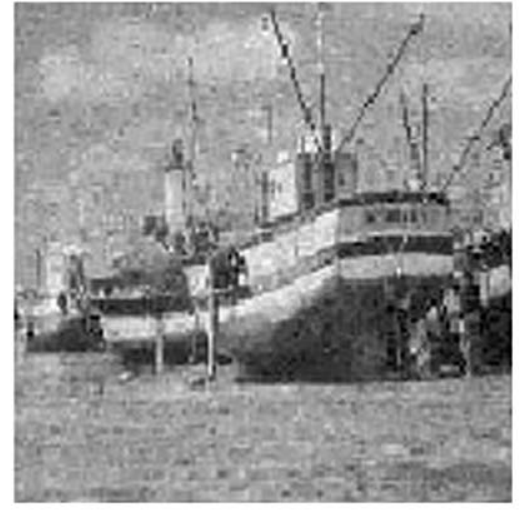

(a)

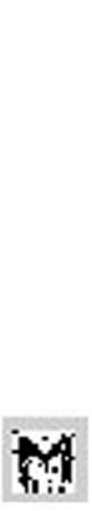

(b)

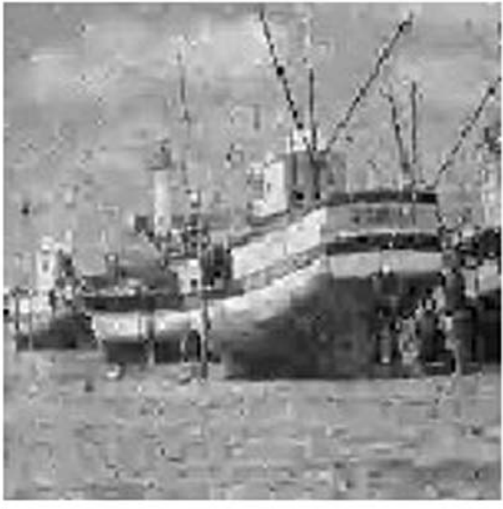

(c)

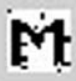

(d)

Fig. 7. (a) Watermarked image without negative modulation and after JPEG compression at quality factor 20; (b) extracted Watermark image from (a), (c) watermarked image using negative modulation and after JPEG compression at quality factor 20; (d) extracted watermark from (c).

operations with $[12,16,17]$ methods. Simulation results show that proposed method always offers better robustness performance compared to other algorithms considering both JPEG and JPEG 2000 compression operations. The watermarking method reported in [17] claims higher robustness against compression operation even at quality factor 10 , but robustness performance against JPEG 2000 compression operation is not reported. Fig. 9(a) and (b) show that the proposed technique can perform better for high compression i.e. low quality image in comparison to the existing techniques $[12,16,17]$. It is also shown that the performance of the proposed technique is also comparable to other methods when low compressed high quality images are taken into consideration.

\section{Conclusions}

The present paper describes a robust block-based digital image watermarking scheme with minimum distortion of the cover image and the algorithm requires low computation cost. The use of both modified entropy model and the conventional Shannon's entropy help to select watermark embedding region for a good compromise between robustness and quality of the watermarked image. As expected, when data are embedded in the medium-informative blocks better resiliency is achieved against the operations like image sharpening, noise addition, dynamic range change of the gray level and compression operation, etc. On the other hand, when data are embedded in the low-informative 


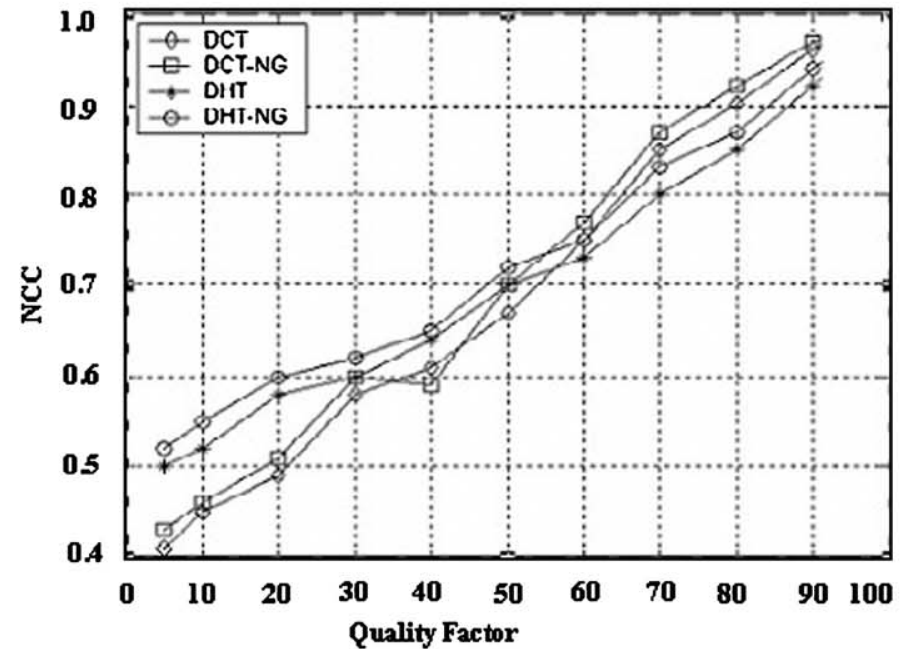

(a)

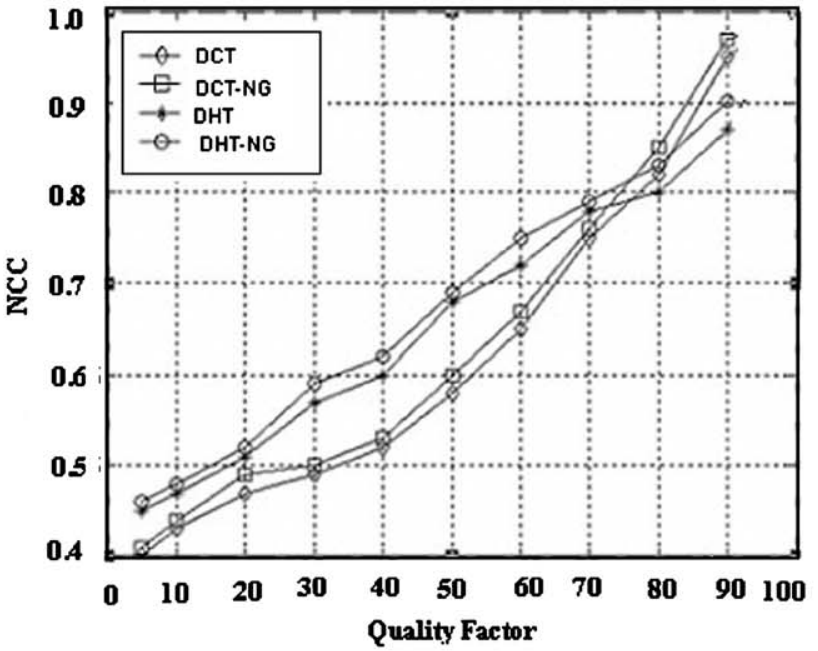

(b)

Fig. 8. Robustness performance of the algorithm against lossy compression operations: (a) JPEG and (b) JPEG 2000; legend DCT-NG, DHT-NG correspond to the result of data embedding in DCT and DHT coefficients after negative modulation.

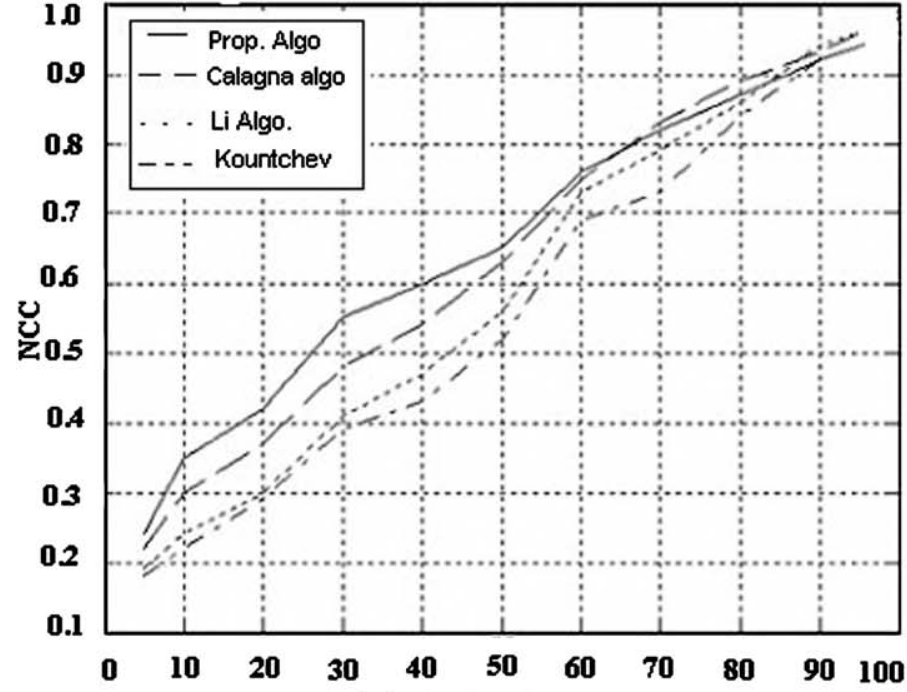

(a) Quality Factor

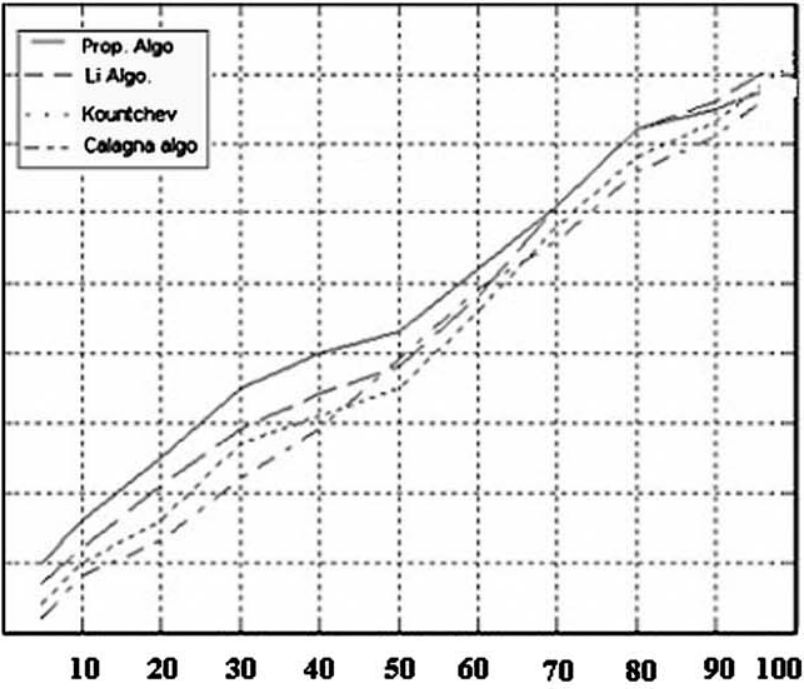

(b) Quality Factor

Fig. 9. Robustness performance of the proposed watermarking method as well as Li, Calagna, and Kountchvev methods against lossy compression operations: (a) JPEG and (b) JPEG 2000.

blocks, better resiliency against smoothing filtering is observed. Adaptive negative modulation further enhances the resiliency against lossy compression. The work is in progress to improve further the robustness efficiency and hardware implementation of the proposed algorithm.

\section{References}

[1] Vleeschouwer CD, Delaigle JF, Macq B. Invisibility and application functionalities in perceptual watermarking-an overview. Proceedings of the IEEE 2002;90:64-77.

[2] Wu M, Liu B. Multimedia data hiding. New York: Springer; 2002.
[3] Arnold M, S M, Wolthusen SD. Digital watermarking and content protection. Boston: Artech House; 2003.

[4] Kutter M, Bhattacharjee SK, Ebrahimi T. Towards second generation watermarking schemes. In: Proceedings of the 6th international conference on image processing, Japan, 1999. p. 320-3.

[5] Nikolaidis A, Pitas I. Region-based image watermarking. IEEE Transaction on Image Processing 2001;10:1726.

[6] Ruanaidh JO, Pun T. Rotation, scale and translation invariant digital image watermarking. In: Proceedings of IEEE ICIP, Atlanta, GA, 1997. p. 536-9.

[7] Ruanaidh JO, Pun T. Rotation, scale and translation invariant spread spectrum digital image watermarking. Signal Processing 1998;66:303-17. 
[8] Cox IJ, Kilian J, LL T, Shamoon T. Secure spread spectrum watermarking for multimedia. IEEE Transaction on Image Processing 1997;6:1673-87.

[9] Podilchuk CI, Zeng W. Image adaptive watermarking using visual models. IEEE Journal on Selected Areas in Communications 1998;16:525-39.

[10] Paquet AH, Ward RK, Pitas I. Wavelet packet-based digital watermarking for image verification and authentication. Signal Processing 2003;83:2117-32.

[11] Ramkumar M, Akansu AN. Capacity estimates for data hiding in compressed images. IEEE Transactions on Image Processing 2001;10:1252-63.

[12] Li H, Wang S, WS, Wen Q. Multiple watermarking using Hadamard transform. In: Lecture Notes in Computer Science, Berlin: Springer; 2005. p. 767-72.

[13] Ho ATS, Shen J, Chow AKK, Woon J. Robust digital image-in-image watermarking algorithm using fast Hadamard transform. In: Proceedings in international symposium on circuits and systems (ISCAS), Atlanta, GA, 2003. p. 826-9.

[14] Gilani SAM, Skodras AN. Watermarking by multiresolution Hadamard transform. In: Proceedings of European conference on electronic imaging and visual arts (EVA 2001), 2001.

[15] Falkowski BJ, Lim LS. Image watermarking using Hadamard transform. Electronics Letters 2000;36:211-3.

[16] Kountchev R, Milanova M, CF, Rubin S. Multimedia watermarking with complex Hadamard transform in the inverse pyramid decomposition. In: Proceedings of IEEE international conference on information reuse and integration, 2003. p. 305-10.

[17] Calagna M, Guo H, Mancini LV, Jajodia S. A robust watermarking system based on SVD compression. In: Proceedings in ACM symposium on applied computing (SAC'06), 2006. p. 1341-7.

[18] Wang Z, Bovik AC, SS HR, Simoncelli EP. Image quality assessment: from error measurement to structural similarity. IEEE Transaction on Image Processing 2004;13:1-14.

[19] Pal NR, Pal SK. Object-back ground segmentation using new definitions of entropy. IEE Proceedings 1989;136:284-95.

[20] Eggers JJ, RB, Girod B. A communication approach to image steganography. In: Proceedings of SPIE 4675 security and watermarking of multimedia contents IV, San Jose, CA, 2002.

[21] Moulin P, Sullivan JAO. Information theoretic analysis of information hiding. IEEE Transactions on Information Theory 2003;49:563-93.

[22] Watson AB, RB, Taylor M. Image quality and entropy masking. In: Proceedings of SPIE conference on human vision, visual processing and digital display VI, 1997.

[23] Maity SP, Kundu MK. Information theoretic approach to spatial image watermarking. In: Proceedings of international conference on computer vision, graphics with special session for young scientists and summer school on image processing (ICCVG-2002), Zakopane, Poland, 2002. p. 524-9.

[24] Maity SP, MKK, Nandi PK. Robust and low cost watermarking using image characteristics. In: Proceedings of fifth international conference on advances in pattern recognition (ICAPR-03), Indian Statistical Institute, Kolkata, India, 2003. p. 351-4.

[25] Shannon CE. A mathematical theory of communication. Bell System Technical Journal 1948;27:379-423.

[26] Gonzalez RC, Woods RE. Digital image processing. New York: Addison-Wesley; 1992.
[27] Fan CP, Yang JF. Fixed-pipeline two-dimensional Hadamard transform algorithms. IEEE Transactions on Signal Processing 1997;45:1669-74.

[28] Alexandrid N, Klinger A. Real-time Walsh-Hadamard transformation. IEEE Transactions on Computers 1972;C21:288-92.

[29] Maity SP, Kundu MK, Maity S. Dual purpose FWT domain spread spectrum image watermarking in real time. Computer and Electrical Engineering 2009;35:415-35.

[30] Sklar B. Digital communication. Englewood Cliffs, NJ: Prentice-Hall; 1988.

[31] Hsu C-T, Wu LJ. Hidden digital watermarks in images. IEEE Transaction on Image Processing 1998;8:58-68.

[32] 〈http://www.cl.cam.ac.uk/fapp2/watermarking〉.

[33] $\langle\mathrm{http}: / /$ sipi.use.edu/services/database/database/html $\rangle$.

[34] 〈http://watermarking.unige.ch/checkmark .

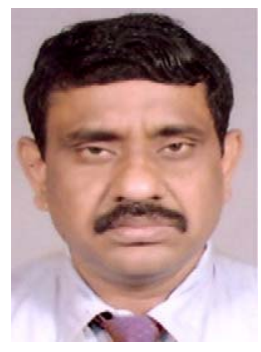

Santi P. Maity received his B.E. in Electronics and Communication Engineering and M.Tech. degree in $\mathrm{Mi}$ crowaves, both from the University of Burdwan, India. He received his Ph.D. degree in Engineering (Computer Science and Technology) from Bengal Engineering and Science University, Shibpur, India. He is at present working as Assistant Professor and Head (Acting) at the Department of Information Technology, Bengal Engineering and Science University, Shibpur. He also worked as Lecturer in Electronics and Telecommunication Engineering Department of the same university from 2000 to 2006. Prior to that, he worked as Lecturer in Electronics and Telecommunication Engineering Department of K.G. Engineering Institute, Bishnupur, Bankura, India and Haldia Institute of Technology, Haldia, India, from 1997 to 2000 . His research areas include digital image watermarking, multiuser detection in CDMA, digital signal processing, digital wireless communication. He delivers several lectures and invited talk on short term course/seminar/workshop/conference and acts as committee member for national and international conferences. He is an Associate Member of Institute of Engineer (India) and a Member of Institute of Electronics and Telecommunication Engineers (IETE), India. He is principal investigator of a project "High Power and Spectral Efficiency Multiuser System for Broadband Wireless Communication" funded by Department of Information Technology, Ministry of Communication and Information Technology, Government of India.

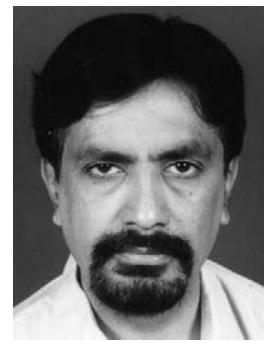

Malay K. Kundu received his B.Tech., M.Tech. and Ph.D. (Tech.) degrees all in Radio physics and Electronics from the University of Calcutta. In 1982, he joined the Indian Statistical Institute, Calcutta, as a faculty member. Currently he is a full professor. He had been the Head of the Machine Intelligence Unit during 1993-1995 and in-charge of the Computer Science Division during 2004-2006. During 1988-1989, he was at the A.I. Laboratory of the Massachusetts Institute of Technology, Cambridge, USA, as a visiting scientist under UN Fellowship program. His 
current research interest include image processing, digital watermarking, content-based image retrieval, fractals, wavelets, soft computing and VLSI design for digital imaging. He received the prestigious VASVIK award for industrial research (Indian equivalent of the Mullard award of UK) in Electronic Sciences and Technology for the year 1999. He also received the Sir J.C. Bose memorial award from Institute of Electronics and Telecommunication Engineers (IETE), India, in the year 1986. He has contributed about 100 research papers in well-known and prestigious archival journals, international refereed conferences and as chapters in monographs and edited volumes. He is the holder of nine US patents, two E.U patents and two international patents and is co-author of the book titled Soft Computing for Image Processing published from Physica-Verlag, Heidelberg. He is fellow of the Indian National Academy of Engineering, India, the National Academy of Sciences, India; Institute of Electronics and Telecommunication Engineers, India. and a senior member of the IEEE. 